\title{
Hans Zwipp
}

Klinik und Poliklinik für Unfall- und Wiederherstellungschirurgie, Universitätsklinikum Carl Gustav Carus, Technische Universität Dresden

\section{Begrüßung zur 4. Dresdner Unfalltagung}

Der Landesverband Bayern und Sachsen der gewerblichen Berufsgenossenschaften und die Klinik für Unfall- und Wiederherstellungschirurgie am Universitätsklinikum „Carl Gustav Carus“ Dresden begrüßen Sie heute, im Frühjahr 2002, zum 4. Mal in Dresden. Als Pendant zur Murnauer Unfalltagung, die bereits seit 1970 in Bayern existiert, ist die biennale Unfalltagung eine feste Veranstaltung in Sachsen geworden. Wir treffen uns heute erstmals in der Aula des Akademiehotels auf dem Gelände des berufsgenossenschaftlichen Instituts für Arbeit und Gesundheit.
Nach Bearbeitung des Problemkreises „Posttraumatische Osteitis“1996, der Darstellung der Thematik „Posttraumatische Korrektureingriffe nach Fehlverheilung an Becken und unterer Extremität" 1998 und der äußerst erfolgreichen Abhandlung der „Frakturen des Fußes" 2000 widmet sich die heutige Tagung dem Thema

\section{„Unterschenkel- und Obere Sprunggelenksfrakturen"}

Diese Thematik steht gerade unter dem Blickwinkel der minimal traumatisierenden chirurgischen Versorgung sowie der noch nicht weit zurückreichenden Einführung von völlig neuen operativen
Konzepten und Implantaten nun auf dem Prüfstand. Im Rahmen der Tagung soll eine Standortbestimmung erfolgen. Mit Ihrer Mithilfe sollen die vorgestellten Konzepte transparenter gemacht sowie kritisch hinterfragt werden.

○) Springer-Verlag 2003

Prof. Dr. Hans Zwipp

Klinik und Poliklinik für Unfallund Wiederherstellungschirurgie, Universitätsklinikum Carl Gustav Carus, Technische Universität Dresden, Fetscherstraße 74,01307 Dresden Tel.: 0351-4583777, Fax: 0351-4584307, E-mail:uwc@rcs.urz.tu-dresden.de
C. Josten

Chirurgische Klinik und Poliklinik, Zentrum für Chirurgie, Universität Leipzig

\section{Die proximale Unterschenkelfraktur: Indikation zur LISS-Osteosynthese}

Autor hat kein Manuskript eingereicht 